\title{
Suicidio en adolescentes y jóvenes en Chile: riesgos relativos, tendencias y desigualdades
}

\author{
Nelson Araneda, ${ }^{1}$ Pablo Sanhueza, ${ }^{2}$ Giovanni Pacheco ${ }^{3}$ y Antonio Sanhueza ${ }^{4}$
}

Forma de citar

Araneda N, Sanhueza P, Pacheco G, Sanhueza A. Suicidio en adolescentes y jóvenes en Chile: riesgos relativos, tendencias y desigualdades. Rev Panam Salud Publica. 2021;45:e4. https://doi.org/10.26633/RPSP.2021.4

RESUMEN

Objetivo. Describir cuantitativamente el riesgo relativo, la tendencia y la desigualdad geográfica del suicidio en adolescentes y jóvenes entre las regiones de Chile, en el periodo 2000 al 2017.

Método. Estudio ecológico poblacional a partir de los registros de defunciones por suicidio. Se estimaron tasas de mortalidad y riesgos relativos (RR) de suicidio por sexo, edad y región. Se estudió la tendencia y desigualdad geográfica del suicidio entre las regiones de Chile; medidas absolutas y relativas de desigualdades geográficas fueron estimadas.

Resultados. Entre 2000 y 2017 se registraron 6292 suicidios en adolescentes y jóvenes en Chile. Las tasas promedio de mortalidad por suicidio en Chile fueron de 8,5; 5,4 y 14.7 por 100000 en los grupos 10-24, 10-19 y 20-24 años, respectivamente, en el periodo 2000-2017. Las tasas más altas de mortalidad por suicidio se encontraron en las regiones de Aisén, Los Lagos, Magallanes y Los Ríos. El riesgo más alto de suicidio se estimó en los hombres $(R R=3,5)$, los jóvenes $(R R=2,7)$ y en la región de Aisén $(R R=2,0)$. La tasa promedio nacional en el grupo 10-24 años se mantuvo en 8,5 por 100000 en los periodos 2000-2008 y 2009-2017. La mayor desigualdad geográfica se encontró en hombres de 20-24 años en el periodo 2000-2008.

Conclusiones. El suicidio en jóvenes y adolescentes de Chile se ha mantenido sin mayores cambios en el periodo de estudio. Los hombres tienen un mayor riesgo de suicidio que las mujeres. Existen desigualdades geográficas entre las regiones de Chile en el suicidio y son más elevadas en hombres de 20 a 24 años. Se recomienda evaluar y fortalecer los programas de prevención del suicidio en los adolescentes y jóvenes, en especial en las regiones y los grupos poblacionales específicos en situación de mayor vulnerabilidad.

Palabras clave Suicidio; adolescente; disparidades en el estado de salud; Chile.

El suicidio se define como el acto en el que una persona deliberadamente se provoca la muerte (1). Este depende de múltiples factores, incluyendo los socioeconómicos, geográficos, culturales y sociales (2), lo cual hace que el suicidio sea difícil de explicar. La Organización Mundial de la Salud (OMS) dio a conocer que el suicidio es una prioridad de salud pública y considerado como una muerte prevenible si se interviene oportunamente por medio de estrategias que sean multisectoriales e integrales (3).
La tasa de suicidio entre adolescentes y jóvenes ha ido en aumento a nivel mundial (4). En el contexto internacional, Europa tuvo el porcentaje más alto de muertes por suicidio, seguida por otras regiones que incluyen la región de las Américas (5). En las Américas, la tasa de suicidio fue de 5,7 por 100 000 en los jóvenes (10-24 años), con tasas de suicidio más altas en los hombres ( 7,7 por 100000$)$ que en las mujeres $(2,4$ por 100 000); los países con las tasas más altas fueron Chile, Ecuador, Guyana, Nicaragua, El Salvador y Surinam (6). Además,

\footnotetext{
Departamento de Educación, Universidad de La Frontera, Temuco, Chile. $\triangle$ nelson.araneda@ufrontera.cl.

2 Departamento de Ingeniería Robótica, Universidad de Maryland, College Park, Estados Unidos de América.
} 3 SEREMI de Salud de La Araucanía, Ministerio de Salud de Chile, Santiago,
Chile.
Organización Panamericana de la Salud, Washington D.C., Estados Unidos de
América. 
el suicidio fue la tercera causa principal de muerte en adolescentes de ambos sexos, mientras que, entre los jóvenes fue la tercera causa principal de muerte en hombres y la cuarta en mujeres $(6,7)$.

Las mujeres de 10-24 años tienen más intentos de suicidio que los hombres (8); sin embargo, los hombres tienen mayor probabilidad de cometer suicidio. Dentro de una gran cantidad de posibles razones que pueden explicar un mayor riesgo de suicidio en los hombres, se encuentra la falta de búsqueda de ayuda, la no aceptación de tratamientos, el aumento del consumo de sustancias narcóticas, comportamientos muy agresivos y el acceso a medios letales (9). Otros factores que pueden estar relacionados al suicidio en los hombres, son los cambios en los roles de género y las diferencias socioeconómicas (9). Además, el suicidio aumenta con la edad al pasar desde la adolescencia a la juventud, lo cual puede ser atribuido al desarrollo cognitivo, los cambios en el desarrollo, la capacidad del individuo para actuar contra sí mismo, el impacto que tienen los períodos críticos y sensibles a lo largo del curso de la vida y su conceptualización de la muerte (10).

El ahorcamiento es el método de suicidio más común en Australia, las Américas, Europa y Japón, mientras que el envenenamiento es el método más común en China e India. Para los intentos de suicidio, los métodos generalmente utilizados involucran productos farmacéuticos o químicos (11-13). En los países de las Américas, los tres métodos principales de suicidio en adolescentes y jóvenes fueron el ahorcamiento, armas de fuego y envenenamiento (6).

Bajos niveles de ingreso y un lento crecimiento económico están asociados con un aumento en las tasas de suicidio (14). La probabilidad de suicidio es mayor en áreas geográficas pobres y con desventajas sociales (factores sociales); además, la probabilidad de suicidio es menor en áreas geográficas con ingresos medios (15). Otras consideraciones debidas al aumento en las tasas de suicidio pueden estar relacionadas con la regulación gubernamental de los medicamentos recetados; el uso de medicamentos no recetados por un médico está asociado con un mayor riesgo de cometer suicidio (16). La falta de políticas y programas dirigidos a la prevención del consumo de sustancias (17) y falta de políticas de salud mental (18), son factores de riesgo que favorecen el incremento del suicidio, especialmente entre adolescentes y jóvenes. Según la Organización Mundial de la Salud (OMS), los trastornos por consumo de alcohol y otras sustancias tienen un peso del 25 a 50\% en todos los suicidios (19). La intoxicación por alcohol puede aumentar la disforia, la disfunción cognitiva, la impulsividad y la intensidad de cometer suicidio. El riesgo de cometer suicidio aumenta a 7 veces después de consumir alcohol; pero si la ingesta es excesiva el aumento se eleva a 37 veces $(20-21)$.

En Chile en el periodo 2000-2011, las tasas más altas de mortalidad por suicidio se encontraron en las regiones de Aisén, Los Lagos y Los Ríos (22). Los tres principales métodos de suicido en hombres fueron ahorcamiento, armas de fuego y envenenamiento, y en mujeres ahorcamiento, envenenamiento y armas de fuego; este mismo patrón se mantuvo en adolescentes y jóvenes (22).

Este estudio tiene como objetivo describir cuantitativamente el riesgo relativo, la tendencia y la desigualdad geográfica del suicidio en adolescentes y jóvenes entre las regiones de Chile, en el periodo 2000 al 2017. Este estudio permitirá obtener información útil para quienes tomen decisiones en la prevención del suicidio en Chile.

\section{MÉTODOS}

El diseño del presente estudio es ecológico, donde las unidades de análisis son las regiones de Chile. Se hizo uso de los registros de defunciones clasificados con los códigos de causa básica de muerte por suicidio (X60-X84 de la CIE-10) en adolescentes y jóvenes de las regiones de Chile, en el periodo 2000 al 2017. Los datos de mortalidad fueron proporcionados por el Departamento de Estadísticas e Información de Salud (DEIS) del Ministerio de Salud de Chile.

Las variables consideradas fueron: grupo de edad (10-24, 15-19, 20-24 años), sexo (ambos sexos, hombre, mujer), región (16 regiones de Chile), y periodo de tiempo (2000-2008, 20092017); esta última variable permite el cálculo de las tasas de suicidio en solo dos periodos de tiempo y no año a año, con objeto de estabilizar las tasas en las regiones. La distribución geográfica de las 16 regiones de Chile corresponden a: i) Norte de Chile: Arica y Parinacota, Tarapacá, Antofagasta y Atacama; ii) Centro de Chile: Coquimbo, Valparaíso, Metropolitana, Libertador Bernardo O'Higgins y Maule; y iii) Sur de Chile: Nuble, Bío-Bío, La Araucanía, Los Ríos, Los Lagos, Aisén y Magallanes y Antártica Chilena.

El análisis exploratorio de los datos consistió en calcular las tasas de mortalidad por suicidio (TMS) y la creación de cuadros y gráficos. Se estimó el riesgo relativo (RR) del suicidio por sexo, grupo de edad y región al ajustar modelos de regresión de Poisson. Para el estudio de las tendencias de la mortalidad por suicidio se utilizaron las fórmulas del cambio porcentual (CP) y el cambio porcentual anual promedio (CPAP) como sigue:

$$
\begin{gathered}
\left.\mathrm{CP}=100^{*}[\mathrm{TMS}(\mathrm{T} 1)-\mathrm{TMS}(\mathrm{T} 0)] / \mathrm{TMS}(\mathrm{T} 0)\right] \mathrm{y} \\
\mathrm{CPAP}=100^{*}[\operatorname{Ln}(\mathrm{TMS}(\mathrm{T} 1))-\operatorname{Ln}(\mathrm{TMS}(\mathrm{T} 0))] /(\mathrm{T} 1-\mathrm{T} 0),
\end{gathered}
$$

donde TMS(T0) y TMS(T1) son los valores de las TMS en los tiempos T0 y T1, respectivamente, y Ln es la función logaritmo natural. Para el estudio de desigualdades geográficas en el suicidio, se estimaron medidas de gradiente: el índice de desigualdad de la pendiente (IDP) como medida absoluta y el índice de desigualdad de la pendiente relativa (IDPR), además de sus intervalos de confianza del 95\% (IC 95\%). A partir de la distribución de las tasas de mortalidad por suicidio en las regiones, ordenadas desde mayor a menor, se obtiene la posición que tiene cada región en el suicidio; esto es obtenido a través del cálculo del Ridit (con valores entre 0 y 1) en cada región del país, el cual corresponde a la posición relativa acumulada de cada región con respecto a su valor poblacional (indicador demográfico). El IDP y IDPR se estiman al ajustar un modelo de regresión lineal:

$$
y=\mathrm{a}+\mathrm{b} \text { Ridit }
$$

donde la variable dependiente y corresponde a la TMS en cada región y la variable independiente es el Ridit en cada región. Se utilizó el método de mínimos cuadrados ponderados para estimar los parámetros de intercepto (a) y pendiente (b) del modelo. El IDP es obtenido al comparar el valor del Ridit $=0$ (región con la tasa más alta) y el valor del Ridit $=1$ 
(región con la tasa más baja), lo cual es equivalente a comparar desde el modelo $y=a+b * 0=a$ con $y=a+b * 1=a+b$. Al realizar la diferencia entre estos dos valores (en términos absolutos) se produce la fórmula IDP $=-b$, y al realizar el cociente entre estos dos valores (en términos relativos) se obtiene la formula del IDPR $=\mathrm{a} /(\mathrm{a}+\mathrm{b})$.

En el presente estudio no fue necesario la revisión por parte de un comité de ética (IRB) ya que se basó en datos agregados de las regiones de Chile que están disponibles públicamente.

\section{RESULTADOS}

\section{Situación general de la mortalidad por suicidio}

En Chile en el periodo 2000 al 2017, se registraron 6292 muertes por suicidio en el grupo poblacional de adolescentes y jóvenes (10-24 años), de las cuales 2676 corresponden al grupo poblacional de adolescentes (10-19 años) y 3616 al grupo poblacional de jóvenes (20-24 años). El número promedio de suicidios anuales en el grupo 10-24 años en el país fue de 350 muertes. Las tasas de mortalidad por suicidio para los grupos 10-24 años, 10-19 años y 20-24 años a nivel nacional fueron estimadas en 8,5; 5,4 y 14,7 por 100000 personas, respectivamente.

En los tres grupos de edades, las tasas más altas de mortalidad para ambos sexos se observaron en las regiones de Aisén, Los Lagos, Magallanes y Los Ríos; y las tasas más bajas en las regiones de Tarapacá, Bío-Bío, Metropolitana y Arica y Parinacota. En el grupo 20-24 años, los valores estimados de las tasas más altas en las cuatro regiones fueron de 23,9, 22,5, 21,6 y 17,8 por 100000 jóvenes, respectivamente, y las tasas de mortalidad más bajas en las cuatro regiones fueron $14,3,13,9,12,5$ y 12,3. En todas las regiones, las tasas de mortalidad en hombres fueron mayores que en mujeres; ver cuadro 1.

\section{Riesgo relativo del suicidio}

En el grupo de 10-24 años, los hombres mostraron un mayor riesgo de mortalidad por suicidio que las mujeres; el riesgo de suicidio en hombres es 3,5 (IC95\% [3,3-3,7]) veces el riesgo en mujeres; ver cuadro 2 . El riesgo de cometer suicidio en jóvenes es 2,7 (IC95\% [2,5-2,9]) veces el riesgo en adolescentes. Por otro lado, el riesgo de suicidio en la región de Aisén es 2,0 (IC95\% $[1,4-2,8])$ veces el riesgo de suicidio en la región de Arica y Parinacota. Patrones similares con RR mayor o igual a 1,5; todos ellos estadísticamente significativos, se observaron en las regiones de Los Lagos (1,9 veces la región de Arica y Parinacota), Magallanes (1,8 veces la región de Arica y Parinacota) y Los Ríos (1,5 veces la región de Arica y Parinacota); ver cuadro 2.

\section{Tendencias de la mortalidad por suicidio}

La figura 1 muestra las tendencias de las tasas de mortalidad por suicidio en Chile para los tres grupos de edades. Se observó que estas tasas se mantuvieron estables entre 2000 y 2007 (tasa mediana de 7,5 muertes por 100 000); luego incrementaron en el periodo 2008 al 2011 (tasa mediana de 10,1 muertes por 100 000); y finalmente, decrecieron en el periodo 2012 al 2017 (tasa mediana de 7,6 muertes por 100000 ).

En el grupo de 10-24 años, la tendencia en la TMS nacional se mantuvo igual en los periodos 2000-2008 y 2009-2017, con un valor estimado de 8,5 suicidios por 100000 para ambos sexos; ver cuadro 3. Las cuatro regiones (de un total de nueve regiones que han disminuido sus tasas en estos dos periodos) que tuvieron una mayor reducción fueron: las regiones de, Aisén, Antofagasta, Nuble y Tarapacá con reducciones del 30,3\%; $28,9 \% ; 26,6 \%$ y $19,9 \%$, respectivamente; la reducción porcentual anual promedio de estas cuatro regiones en los dos periodos

CUADRO 1. Tasas de mortalidad por suicidio para los tres grupos de edades y por sexo en Chile y sus 16 regiones, en el periodo 2000-2017.

\begin{tabular}{|c|c|c|c|c|c|c|c|c|c|}
\hline \multirow{2}{*}{$\begin{array}{c}\text { Lugar Geográfico/edad } \\
\text { País/Regiones }\end{array}$} & \multicolumn{3}{|c|}{10 a 24 años } & \multicolumn{3}{|c|}{10 a 19 años } & \multicolumn{3}{|c|}{20 a 24 años } \\
\hline & Total & Hombre & Mujer & Total & Hombre & Mujer & Total & Hombre & Mujer \\
\hline Chile & 8,5 & 13,1 & 3,8 & 5,4 & 7,6 & 3,1 & 14,7 & 24,2 & 5,0 \\
\hline Región de Arica y Parinacota & 7,0 & 10,3 & 3,4 & 4,4 & 5,7 & 3,0 & 12,3 & 19,8 & 4,2 \\
\hline Región de Tarapaca & 8,0 & 12,5 & 3,1 & 4,9 & 7,1 & 2,5 & 14,3 & 23,3 & 4,3 \\
\hline Región de Antofagasta & 8,6 & 12,8 & 4,1 & 5,7 & 8,0 & 3,2 & 14,6 & 22,3 & 6,0 \\
\hline Región de Atacama & 10,0 & 16,2 & 3,4 & 6,7 & 10,4 & 2,8 & 17,3 & 29,0 & 4,7 \\
\hline Región de Coquimbo & 8,4 & 12,6 & 4,2 & 5,1 & 6,9 & 3,2 & 15,5 & 24,7 & 6,1 \\
\hline Región de Valparaíso & 9,2 & 13,7 & 4,4 & 5,9 & 8,3 & 3,4 & 15,5 & 24,2 & 6,4 \\
\hline Región Metropolitana & 7,2 & 11,0 & 3,3 & 4,5 & 6,2 & 2,7 & 12,5 & 20,3 & 4,5 \\
\hline $\begin{array}{l}\text { Región Libertador Bernardo } \\
\text { O'Higgins }\end{array}$ & 8,9 & 13,5 & 4,0 & 5,7 & 8,1 & 3,2 & 16,3 & 26,1 & 6,1 \\
\hline Región del Maule & 9,2 & 13,8 & 4,4 & 5,3 & 7,5 & 3,0 & 17,6 & 27,9 & 7,2 \\
\hline Región de Ñuble & 8,6 & 12,5 & 4,6 & 6,1 & 7,8 & 4,4 & 14,1 & 23,1 & 5,0 \\
\hline Región del Bío-Bío & 7,8 & 12,5 & 3,1 & 4,9 & 7,2 & 2,5 & 13,9 & 23,5 & 4,3 \\
\hline Región de La Araucania & 9,6 & 15,9 & 3,1 & 6,2 & 9,2 & 3,1 & 16,8 & 30,6 & 3,1 \\
\hline Región de Los Ríos & 10,4 & 16,0 & 4,6 & 6,9 & 9,4 & 4,3 & 17,8 & 29,9 & 5,1 \\
\hline Región de Los Lagos & 13,4 & 20,9 & 5,5 & 9,3 & 12,6 & 5,7 & 22,5 & 38,9 & 5,1 \\
\hline Región de Aisén & 14,0 & 22,0 & 5,0 & 9,8 & 14,1 & 5,1 & 23,9 & 40,1 & 4,7 \\
\hline $\begin{array}{l}\text { Región Magallanes y Antártica } \\
\text { Chilena }\end{array}$ & 12,5 & 19,2 & 4,5 & 8,0 & 10,8 & 4,7 & 21,6 & 35,3 & 4,1 \\
\hline
\end{tabular}


CUADRO 2. Estimación del riesgo relativo (RR), intervalo de confianza del $95 \%$ y sus valor $p$, de las variables sexo (mujer como referencia), grupo de edad (10-19 años como referencia) y región (Arica y Parinacota como referencia).

\begin{tabular}{|c|c|c|c|c|c|}
\hline \multicolumn{2}{|c|}{ Variables } & \multirow{2}{*}{$\begin{array}{l}\text { RR } \\
3,5\end{array}$} & \multirow{2}{*}{$\begin{array}{c}\text { Lim Inf } \\
3,3\end{array}$} & \multirow{2}{*}{$\begin{array}{l}\text { Lim Sup } \\
\qquad 3,7\end{array}$} & \multirow{2}{*}{$\begin{array}{l}\text { Valor-p } \\
<.0001\end{array}$} \\
\hline Sexo & Hombre vs Mujer & & & & \\
\hline $\begin{array}{l}\text { Grupo de } \\
\text { Edad }\end{array}$ & $\begin{array}{l}\text { 20-24 años vs 10-19 } \\
\text { años }\end{array}$ & 2,7 & 2,5 & 2,9 & $<.0001$ \\
\hline \multirow[t]{16}{*}{ Region } & Aisén & 2,0 & 1,4 & 2,8 & $<.0001$ \\
\hline & Los Lagos & 1,9 & 1,5 & 2,5 & $<.0001$ \\
\hline & $\begin{array}{l}\text { Magallanes y Antártica } \\
\text { Chilena }\end{array}$ & 1,8 & 1,3 & 2,5 & 0.0005 \\
\hline & Los Ríos & 1,5 & 1,1 & 2,0 & 0.006 \\
\hline & Atacama & 1,4 & 1,1 & 1,9 & 0.0221 \\
\hline & La Araucanía & 1,4 & 1,0 & 1,8 & 0.0207 \\
\hline & Valparaíso & 1,3 & 1,0 & 1,7 & 0.0405 \\
\hline & Maule & 1,3 & 1,0 & 1,7 & 0.0469 \\
\hline & $\begin{array}{l}\text { Libertador Bernardo } \\
\text { O'Higgins }\end{array}$ & 1,3 & 1,0 & 1,7 & 0.0835 \\
\hline & Antofagasta & 1,2 & 0,9 & 1,6 & 0.1493 \\
\hline & Ñuble & 1,2 & 0,9 & 1,6 & 0.1642 \\
\hline & Coquimbo & 1,2 & 0,9 & 1,6 & 0.194 \\
\hline & Tarapaca & 1,1 & 0,8 & 1,5 & 0.4226 \\
\hline & Bío-Bío & 1,1 & 0,9 & 1,4 & 0.4011 \\
\hline & Metropolitana & 1,0 & 0,8 & 1,3 & 0.8142 \\
\hline & $\begin{array}{l}\text { Arica y Parinacota } \\
\text { (referencia) }\end{array}$ & - & NA & NA & NA \\
\hline
\end{tabular}

fue del $4,0 \%, 3,8 \%, 3,4 \%$ y 2,5\%, respectivamente (cuadro 3 ). Por otro lado, las cuatro regiones (de un total de siete regiones que incrementaron sus tasas entre estos dos periodos) con mayores aumentos de las tasas en los dos periodos fueron: las regiones de Los Ríos, Coquimbo, La Araucanía y Atacama, con porcentajes de aumento del 10,9\%, 11,8\%, 13,0\% y 13,0\%, respectivamente (cuadro 3 ).

\section{Desigualdades Geográficas del suicidio}

Para el grupo 10-24 años en ambos sexos, la desigualdad geográfica entre las regiones de Chile (absoluta y relativa) y la tasa nacional se mantuvieron constantes en los periodos de tiempo (cuadro 4). Sin embargo, para el grupo 10-19 años en ambos sexos, las desigualdades geográficas aumentaron en los dos periodos de tiempo, aunque la tasa nacional se mantuvo constante. En el grupo de 20-24 años y en ambos sexos, la desigualdad y la tasa nacional disminuyeron en el tiempo; ver cuadro 4. Un patrón similar se presentó en el comportamiento de la desigualdad geográfica y la tasa nacional para los tres grupos (10-24 años, 10-19 años y 20-24 años) en hombres. Para cada uno de los grupos en mujeres, la desigualdad disminuyó en el tiempo, pero la tasa nacional aumento levemente; ver cuadro 4.

En general, se observa que se produce mayor desigualdad geográfica en las tasas de mortalidad por suicidio en hombres que en mujeres; esto se presentó en los tres grupos; además, estas diferencias en la desigualdad absoluta son estadísticamente significativas (los IC95\% no se superponen); ver cuadro
4. Por ejemplo, considerando el grupo 20-24 años, el IDP [IC $95 \%$ ] en hombres fue de $18,1[14,4-21,9]$ y en mujeres 5,1 [4,26,0], en el periodo 2000-2008.

La mayor desigualdad geográfica se presenta en la TMS en el grupo 20-24 años en hombres, con valores estimados del IDPA = 19,1 y IDPR = 2,1 en el periodo 2000-2008; estos valores indican que, en términos absolutos, entre la región con la TMS más alta y la región con la TMS más baja existe un exceso de mortalidad equivalente a 19 suicidios por 100000 jóvenes. En términos relativos, el riesgo de suicidio en la región con la tasa de mortalidad más alta es dos veces el riesgo de suicidio en la región con la tasa de mortalidad más baja. Por otro lado, se obtuvo un valor estimado del IDP=14,1 y IDPR=1,9 en el periodo 2009-2017, es decir tanto la desigualdad absoluta como la relativa disminuyeron en el tiempo; además, la tasa nacional disminuyó en el mismo periodo (cuadro 4). Este patrón en la disminución de la tasa nacional y la desigualdad es el mejor escenario que se debería esperar.

\section{DISCUSIÓN}

Similar a lo encontrado en otros estudios $(6,22,23)$, la mortalidad por suicidio en las regiones de Chile y a nivel nacional fue mayor en los jóvenes que en los adolescentes, y mayor entre hombres que en mujeres. Esto indica que es importante realizar esfuerzos de prevención del suicidio en edades tempranas. Además, se debería disponer de programas de prevención diferenciados para hombres y mujeres (24). Por otro lado, se encontró que existen marcadas desigualdades geográficas en la TMS en los adolescentes y jóvenes, donde el riesgo en la región con la TMS más alta es 2 veces el riesgo de la región con la TMS más baja en el periodo 2000-2008. La Región de Aisén tuvo la TMS más alta en este periodo. En Aisén se llevó a cabo un programa comunitario denominado Red para la Atención y Derivación de Adolescentes en Riesgo Suicida (RADAR) (25). Es interesante mencionar que Aisén fue una de las tres regiones con mayores reducciones en sus TMS en los adolescentes y jóvenes, alcanzando el $29 \%$ de reducción entre los periodos 2000-2008 y 2009-2017. Se ha demostrado que intervenciones de prevención de la conducta suicida en establecimientos educacionales resultan ser muy efectivas en el aumento de factores protectores del suicidio, como también la disminución de factores de riesgo (26). Una intervención muy efectiva para la prevención del suicidio en los adolescentes y jóvenes es la distribución de información sobre la conducta suicida a toda la comunidad educativa (incluyendo los padres), donde se muestre como y donde obtener ayuda para evitar el suicidio (26). El riesgo de suicidio en personas con enfermedades mentales es 10 veces el riesgo en personas sin este tipo de enfermedades (27). Se ha recomendado que los establecimientos educacionales consideren metodologías de prevención del suicidio que contengan recursos atractivos, dinámicos y didácticos, incluyendo recursos audiovisuales (28).

Bajo el liderazgo del Ministerio de Salud y con la colaboración del Ministerio de Educación de Chile, se desarrollaron recomendaciones para desplegar acciones preventivas del suicidio en adolescentes y jóvenes en establecimientos educacionales del país (29). Estas acciones están dirigidas al personal para detectar tempranamente y manejar adecuadamente las señales de alerta del suicidio, además de tener acceso a los servicios de salud. Estas recomendaciones forman parte del Programa Nacional de 
Figura 1. Tendencias de la mortalidad por suicio por los grupos de edades en el periodo 2000 al 2017.

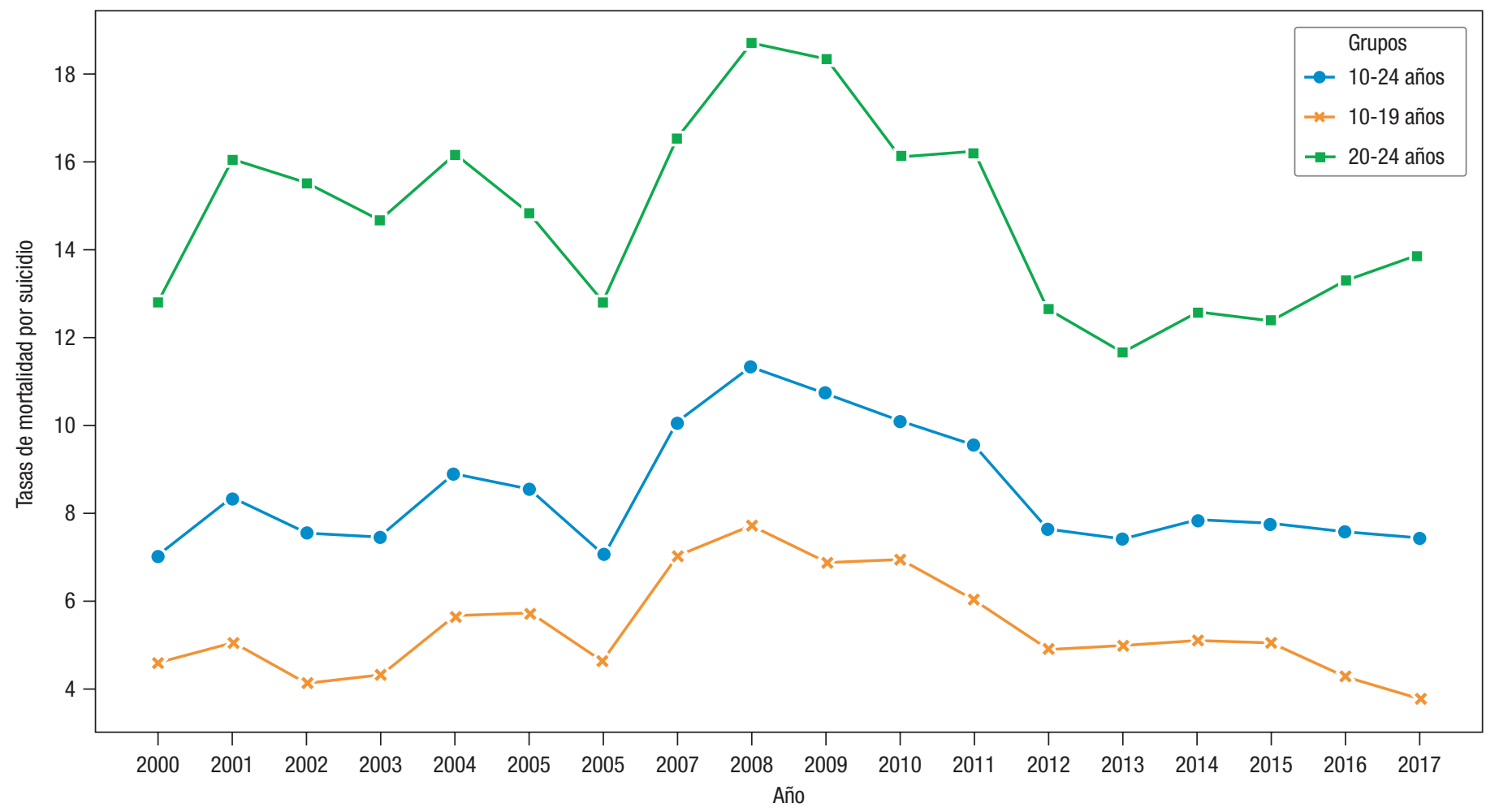

Prevención del Suicidio en Chile (30), el cual utiliza un modelo de intervención basado en la intersectorialidad y está dirigido a las regiones del país. Es importante que el programa nacional de prevención del suicidio dirigido a los adolescentes y jóvenes de Chile pueda ser evaluado continuamente y particularmente en las regiones que presentan mayores TMS. Esto permitirá fortalecer y mejorar el programa de prevención de suicidio en las regiones y disminuir las TMS (3).

La Estrategia Nacional de Salud para la década 2011-2020 del Ministerio de Salud de Chile incluyó una meta nacional de reducción del 15\% en la TMS en adolescentes entre el 2011 y 2020 (31). Esta meta nacional no consideró la variabilidad de las TMS que existen entre las regiones del país. La OPS recomienda el establecimiento de metas conjuntas en el promedio nacional y en la desigualdad geográfica de indicadores de salud (32). Se recomienda que el nuevo plan estratégico del Ministerio de Salud de Chile pueda incorporar metas explicitas en la reducción de las desigualdades geográficas de las TMS en los adolescentes y jóvenes. Además, es relevante conocer las características de las intervenciones exitosas de prevención del suicidio que se han llevado a cabo en ciertas regiones del país, las cuales pueden servir en otras regiones, como también conocer la disminución en el número de casos hospitalizados por intento de suicidio en cada región.

Se deben fortalecer los sistemas de información y vigilancia del suicidio en las regiones de Chile. Los datos deben ser desagregados por estratificadores tales como: edad, sexo, etnia, orientación sexual, nivel socioeconómico, escolaridad, etc. Esto permitirá describir las desigualdades sociales del suicido en el país y en las regiones, con el objeto de intervenir en grupos poblaciones en situación de mayor vulnerabilidad social y posibilitar la disminución del suicidio (33). La orientación sexual e identidad de género son considerados condiciones de riesgo para el suicido (34), por ello es necesario desarrollar investigaciones sobre esta problemática de salud en los grupos poblacionales de adolescentes y jóvenes LGBT. Se recomienda incluir preguntas sobre orientación sexual e identidad de género en la recolección de datos que se obtienen en los sistemas de información y vigilancia del suicidio en los adolescentes y jóvenes de las regiones de Chile, así como también la implementación de estudios que permitan describir los aspectos de la vida de las personas LGBT relacionados con problemáticas de salud mental y llevar a cabo intervenciones en adolescentes y jóvenes LGBT.

El suicidio entre adolescentes y jóvenes en Chile se ha mantenido estable entre los periodos 2000-2008 y 2009-2017; sin embargo, ha habido un aumento en algunas regiones del país. Estudios recomiendan que el desarrollo y cumplimiento de las leyes de protección y políticas adecuadas, posibilitan la reducción de factores de riesgo y las tasas de suicidio entre los jóvenes. Se ha mostrado una disminución en la tasa de suicidio por armas de fuego, en los jóvenes, cuando se implementa el cumplimiento de una política de limitación en el control de éstas $(4,35)$. Es necesario contar con nuevos estudios cualitativos que permitan investigar las características relacionadas con el suicidio en los adolescentes y jóvenes. Se recomienda llevar a cabo estudios longitudinales para determinar la causalidad y los factores contextuales que influyen en las conductas suicidas.

Equipos multidisciplinarios forman parte del programa de prevención del suicidio en Chile, lo cual permite dar respuesta más eficaz a la salud y el desarrollo de los adolescentes y jóvenes. Los proveedores de servicios de salud, profesionales de 
CUADRO 3. Tasas de mortalidad por suicidio, cambio porcentual (CP \%) y cambio porcentual anual promedio (CPAP \%) entre los periodos 2000-2008 y 2009-2017 por grupo de edad (10-24 años, 10-19 años y 20-24 años).

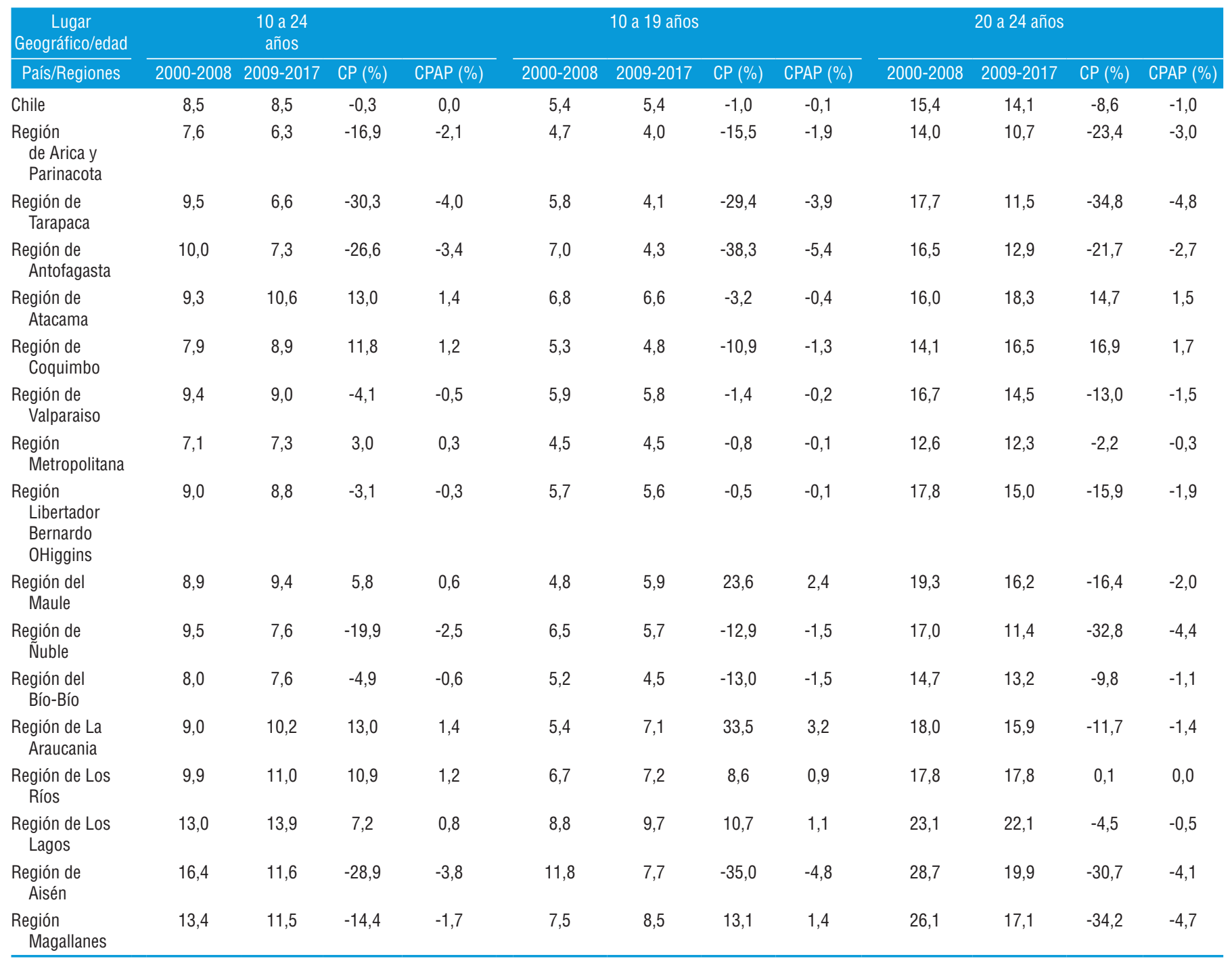

establecimientos educacionales, profesores universitarios y promotores de salud comunitaria, entre otros, son pilares fundamentales para mejorar la salud mental de los adolescentes y jóvenes, y ayudar a prevenir el suicidio. Por otro lado, campañas mediante redes sociales, educativas y uso de medios de comunicación masivos, permiten la sensibilización de este fenómeno en adolescentes y jóvenes y en la población en general, favoreciendo la prevención del suicidio (36); esto permite que las personas puedan hablar abiertamente sobre el suicidio, lo cual ayuda a la prevención de este fenómeno (3).

Involucrar a los adolescentes y jóvenes en la evaluación de los programas existentes de prevención del suicidio, permitirá que ellos reflejen mejor sus necesidades y resulten ser programas más pertinentes. Es posible construir regiones más seguras contra el suicidio al promover la educación y prevención como un componente de la salud pública regional, así como la salud mental y bienestar de los adolescentes y jóvenes. Se ha demostrado que intervenciones conjuntas entre los adolescentes y sus padres han producido una reducción en los factores de riesgo y un aumento en los factores de protección del suicidio (37).

Una fortaleza del estudio es la inclusión de medidas de desigualdades geográficas en el suicidio. Limitaciones del estudio tienen que ver con el subregistro y mala clasificación de las muertes; un ejemplo de mala clasificación es considerar un suicidio como una muerte por accidente de tránsito o como una causa indeterminada.

Grupos poblaciones en situación de mayor vulnerabilidad social, tales como: sin empleo, dependientes de sustancias nocivas, sin acceso a los servicios de apoyo psicológico y psiquiátrico, pueden tener mayores posibilidades de experimentar suicidio, considerando que estos son factores de riesgo relevantes. Esto deja de manifiesto la importancia de llevar a cabo investigaciones sobre los factores sociales, económicos y culturales que afectan a los adolescentes y jóvenes con mayor grado de vulnerabilidad (incluyendo grupos indígenas) y su 
CUADRO 4. Estimaciones del índice desigualdad de la pendiente (IDP) y del índice desigualdad de la pendiente relativa (IDPR) y la tasa nacional por grupo de edad y sexo en los periodos 2000-2008 y 2009-2017.

\begin{tabular}{|c|c|c|c|c|c|c|c|c|c|c|}
\hline $\begin{array}{c}\begin{array}{c}\text { Variables/ } \\
\text { edad }\end{array} \\
\text { Sexo }\end{array}$ & $\begin{array}{l}\text { Periodo de } \\
\text { Tiempo }\end{array}$ & \multicolumn{3}{|c|}{$\begin{array}{c}10 \text { a } 24 \\
\text { años }\end{array}$} & \multicolumn{3}{|c|}{$\begin{array}{c}10 \text { a } 19 \\
\text { años }\end{array}$} & \multicolumn{3}{|c|}{$\begin{array}{c}20 \text { a } 24 \\
\text { años } \\
\end{array}$} \\
\hline & 2009-2017 & $\begin{array}{c}5,2 \\
{[3,8-6,7]}\end{array}$ & $\begin{array}{c}1,9 \\
{[1,5-2,9]}\end{array}$ & 8,5 & $\begin{array}{c}4,2 \\
{[3,0-5,5]}\end{array}$ & $\begin{array}{c}2,3 \\
{[1,6-5,3]}\end{array}$ & 5,4 & $\begin{array}{c}8,1 \\
{[6,2-10,1]}\end{array}$ & $\begin{array}{c}1,8 \\
{[1,5-2,4]}\end{array}$ & 14,1 \\
\hline Hombres & 2009-2017 & $\begin{array}{c}9,0 \\
{[6,7-11,3]}\end{array}$ & $\begin{array}{c}2,1 \\
{[1,6-3,4]}\end{array}$ & 12,9 & $\begin{array}{c}7,0 \\
{[5,5-8,6]}\end{array}$ & $\begin{array}{c}2,8 \\
{[1,9-6,7]}\end{array}$ & 7,5 & $\begin{array}{c}14,1 \\
{[10,2-18,1]}\end{array}$ & $\begin{array}{c}1,9 \\
{[1,5-2,9]}\end{array}$ & 22,7 \\
\hline \multirow[t]{2}{*}{ Mujeres } & $2000-2008$ & $\begin{array}{c}3,2 \\
{[2,6-3,8]}\end{array}$ & $\begin{array}{c}2,6 \\
{[1,9-4,5]}\end{array}$ & 3,6 & $\begin{array}{c}3,0 \\
{[2,0-4,0]}\end{array}$ & $\begin{array}{c}2,9 \\
{[1,6-6,9]}\end{array}$ & 3,1 & $\begin{array}{c}5,1 \\
{[4,2-6,0]}\end{array}$ & $\begin{array}{c}3,4 \\
{[2,1-9,9]}\end{array}$ & 4,7 \\
\hline & 2009-2017 & $\begin{array}{c}2,4 \\
{[2,0-2,8]}\end{array}$ & $\begin{array}{c}1,9 \\
{[1,6-2,4]}\end{array}$ & 3,9 & $\begin{array}{c}2,0 \\
{[1,3-2,8]}\end{array}$ & $\begin{array}{c}1,9 \\
{[1,4-3,8]}\end{array}$ & 3,2 & $\begin{array}{c}4,7 \\
{[3,8-5,6]}\end{array}$ & $\begin{array}{c}2,6 \\
{[1,9-4,8]}\end{array}$ & 5,2 \\
\hline
\end{tabular}

asociación con el suicidio; todo esto con la meta de establecer políticas y programas de prevención del suicidio en grupos poblaciones en situación de mayor vulnerabilidad (38).

Contribución de autores. NA, PS, GP y AS desarrollaron el estudio original, analizaron los datos, y escribieron y revisaron el manuscrito. Todos los autores revisaron y aprobaron la versión final del manuscrito.

Agradecimientos. Nelson Araneda agradece la deferencia y apoyo de sus colegas del Departamento de Educación y del Consejo de la Facultad de Educación, Ciencias Sociales y
Humanidades de la Universidad de La Frontera por permitirle llevar a cabo un año sabático, durante el cual fue desarrollado el presente artículo científico.

Financiación. El presente estudio no ha recibido financiación externa.

Conflicto de intereses. Ninguno declarado por los autores.

Declaración. Las opiniones expresadas en este manuscrito son responsabilidad de los autores y no reflejan necesariamente los criterios ni la política de la RPSP/PAJPH y / o de la OPS.

\section{REFERENCIAS}

1. Kolves K, De Leo D. Regions with the Highest Suicide Rates for Children and Adolescents - Some Observations. J Child Adolesc Behav. 2014;2(2):1000e104.

2. Goldsmith SK, Pellmar TC, Kleinman AM et al, eds. Reducing Suicide: A National Imperative. Washington, DC: National Academies Press; 2002.

3. Organización Mundial de la Salud. Prevención del suicidio: un imperativo global; Washington, DC: OPS, 2014.

4. Viner RM, Coffey C, Mathers C et al. 50-year mortality trends in children and young people: a study of 50 low-income, middle-income, and high-income countries. Lancet 2011; 377: 1162-74

5. Patton GC, Coffey C, Sawyer S, et al. Global Patterns of Mortality in Young People: A Systematic Analysis of Population Health Data. Lancet 2009; 374 (9693): 881-892.

6. Quinlan-Davidson M, Sanhueza A, Espinosa I, Escamilla-Cejudo JA, Maddaleno M. Suicide among young people in the Americas. J Adolesc Health. 2014;54(3):262-8.

7. Pitman A, Krysinska K, Osborn D et al. Suicide in Young Men. Lancet 2012, 379:2383-2392.

8. Cantor C. Suicide in the Western world. In Hawton K \& van Heeringen $\mathrm{K}$ (Eds.), International handbook of suicide and attempted suicide. Chichester: Wiley; 2000

9. Mittendorfer-Rutz E. Trends of youth suicide in Europe during the $1980 \mathrm{~s}$ and 1990s - gender differences and implications for prevention. JMHG 2006; 3(3): 250-257.

10. Bingham CR, Bennion LD, Openshaw DK, et al. An analysis of age, gender and racial differences in recent national trends of youth suicide. J Adolesc 1994;17:53e71.
11. Morrell S, Page AN, Taylor RJ. The decline in Australian young male suicide. Soc Sci Med 2007; 64: 747-54.

12. Varnik A, Kolves K, Alllik J, et al. Gender issues in suicide rates, trends and methods among youths aged 15-24 in 15 European countries. J Affect Disord 2009; 113: 216-26.

13. Ojima T, Nakamura Y, Detels R. Comparative study about methods of suicide between Japan and the United States. J Epidemiol 2004; 14: 187-92.

14. Lester D, Yang BJ. Microsocioeconomics versus macrosocioeconomics as a model for examining suicide. Psychol Rep 1991; 69: 735-8.

15. Rehkopf DH, Buka SL. The association between suicide and the socio-economic characteristics of geographical areas: a systematic review. Psychological medicine. 2006 Feb;36(2):145-57.

16. Buckley NA, Whyte IM, Dawson AH et al. Correlations between prescriptions and drugs taken in self-poisoning. Implications for prescribers and drug regulation. The Medical Journal of Australia 1995; 162(4):194-197.

17. Colucci E \& Martin G. Ethnocultural aspects of suicide in young people: a systematic literature review part 2: risk factors, precipitating agents, and attitudes toward suicide. Suicide and Life-Threatening Behavior 2007; 37(2): 222-237.

18. Evans E, Hawton K, \& Rodham K. Factors associated with suicidal phenomena in adolescents: A systematic review of population-based studies. Clinical Psychology Review 2004; 24: 957-979.

19. Schneider B. Substance use disorders and risk for completed suicide. Arch Suicide Res. 2009; 13(4): 303-16. 
20. World Health Organization. 2018 Global status report on alcohol and health 2018. World Health Organization. Disponible en: https://apps.who.int/iris/handle/10665/274603

21. Borges G, Bagge CL, Cherpitel CJ, Conner KR, Orozco R, Rossow I (2017). A metaanalysis of acute use of alcohol and the risk of suicide attempt. 47(5):949-57.

22. Otzen T, Sanhueza A, Manterola C, Escamilla-Cejudo JA. Mortalidad por suicidio en Chile: tendencias en los años 1998-2011. Rev Med Chile 2014; 142: 305-313.

23. Gerstner RMF, Soriano I, Sanhueza A, Caffe S, Kestel D. Epidemiología del suicidio de adolescentes y jóvenes en Ecuador. Rev Pan Am Salud Publica. 2018;42:e100.

24. Sánchez-Teruel, D., \& Robles-Bello, M. (2015). Variables demográficas y psicosociales que modulan el riesgo de suicidio en adolescentes. Revista INFAD de Psicología. International Journal of Developmental and Educational Psychology, 1(1), 579-590.

25. F. Bustamante Francisco, Urquidi Cinthya, Florenzano Ramón, Barrueto Carolina, Hoyos Jaime de los, Ampuero Karla et al. El programa RADAR para la prevención del suicidio en adolescentes de la región de Aysén, Chile: resultados preliminares. Rev Chil Pediatr. 2018;89(1):145-148 DOI: 10.4067/S0370-41062018000100145

26. Irarrázaval, M., Martínez, V., Behn, A., \& Martínez, P. Revisión sistemática de estudios de efectividad, costo-efectividad, y programas de salud en establecimientos educacionales destinados a la prevención de conductas suicidas en adolescentes. Licitación ID 757-69-L116. Santiago, Chile: Subsecretaría de Salud Pública, Ministerio de Salud; 2017

27. Vijayakumar, Lakshmi; Nagaraj, K.; Sujit Jhon. Suicide and suicide prevention in developing countries, Desease Control Priorities Project. Working Paper $\mathrm{N}^{\circ}$ 27, June 2004

28. Surgenor, P., Quinn, P., \& Hughes, C. (2016). Ten Recommendations for Effective School-Based, Adolescent, Suicide Prevention Programs. School Mental Health, 1-12. doi:10.1007/s12310-0169189-9

29. Ministerio de Salud de Chile. Recomendaciones para la Prevención de la Conducta Suicida en Establecimientos Educacionales. Programa Nacional de Prevención de Suicidio; 2019.Santiago: Ministerio de Salud de Chile;2019. Disponible en: https:/ / www.minsal. cl/wp-content/uploads/2019/06/2019.05.15_PREVENCION-SUICIDIO-EN-ESTABLECIMIENTOS-EDUCACIONALES-web.pdf
30. Ministerio de Salud de Chile. Programa Nacional de Prevención del Suicidio: Orientaciones para su implementación: MINSAL, 2013.

31. Ministerio de Salud de Chile. Estrategia Nacional de Salud para el cumplimiento de los Objetivos Sanitarios de la década 2011-2020; Santiago: Ministerio de Salud de Chile;2010.

32. Sanhueza, Espinosa, Mujica y Barbosa. Sin dejar a nadie atrás: una metodologia para establecer metas de reduccion de desigualdad en salud del ODS3. Rev Panam Salud Publica. 2020;44:e155. https:// doi.org/10.26633/RPSP.2020.155

33. Mujica OJ, Moreno CM. De la retórica a la acción: medir desigualdades en salud para "no dejar a nadie atrás". Rev Panam Salud Publica. 2019;43:e12.

34. Haas A, Eliason M, Mays V, Mathy R, Cochran S, Clayton P, et al. Suicide and suicide risk in lesbian, gay, bisexual, and transgender populations: Review and recommendations. Journal Of Homosexuality 2011; 58 (1): 10-51.

35. Cantor $\mathrm{CH}$, Slater PJ. The impact of firearm control legislation on suicide in Queensland, preliminary findings. Med J Australia 1995; $162: 583 \mathrm{e} 5$

36. Niederkrotenthaler T, Till B, Herberth A, et al. Can media effects counteract legislation reforms? The case of adolescent firearm suicides in the wake of the Austrian firearm legislation. J Adolesc Health 2009;44:90e3.

37. Centre for Suicide Prevention. Straight talk: Youth suicide prevention workshop, Calgargy, Canada; 2012. Disponible en: http://suicideinfo.ca/Training/WorkshopDescriptions. Acceso el 17 noviembre 2020.

38. McPhedran S, Baker J. Suicide prevention and method restriction: Evaluating the impact of limiting access to lethal means among young Australians. Arch Suicide Res 2012;16:135e46.

Manuscrito recibido el 30 de septiembre de 2020. Aceptado para su publicación, tras revisión, el 19 de noviembre de 2020. 


\section{Suicide in adolescents and young adults in Chile: relative risks, trends, and inequalities}

ABSTRACT Objective. Quantitatively describe relative risk, trends, and geographical inequalities in suicide in adolescents and young adults in regions of Chile, from 2000 to 2017.

Methodology. Ecological population study based on records of death by suicide. Suicide death rates and relative risk (RR) were estimated, by sex, age, and region. Trends and geographical inequalities in suicide in regions of Chile were studied; absolute and relative measures of geographical inequalities were estimated.

Results. Between 2000 and 2017, there were 6,292 suicides in adolescents and young adults in Chile. The average rates of death by suicide in Chile were 8.5, 5.4, and 14.7 per 100,000 in the 10-24, 10-19, and 20-24year age groups, respectively, in the period 2000-2017. The highest suicide death rates were found in the Aisén, Los Lagos, Magellan and Los Ríos regions. The highest risk of suicide was estimated in men $(R R=3.5)$, young adults $(R R=2.7)$, and the Aisén region $(R R=2.0)$. The national average rate in the 10-24 age group remained at 8.5 per 100,000 in the periods $2000-2008$ and $2009-2017$. The greatest geographical inequality was found in men 20-24 years old in the period 2000-2008.

Conclusions. Suicide in young adults and adolescents in Chile remained unchanged in the study period. Men have a higher risk of suicide than women. There are geographical inequalities in suicide between Chile's regions and they are highest in men between ages 20 and 24. It is recommended to evaluate and strengthen suicide prevention programs in adolescents and young adults, especially in the most vulnerable regions and population groups.

Keywords $\quad$ Suicide; adolescent; health status disparities; Chile.

\section{Suicídio em adolescentes e jovens no Chile: riscos relativos, tendências e desigualdades}

RESUMO Objetivo. Descrever quantitativamente o risco relativo, tendência e desigualdades geográficas do suicídio em adolescentes e jovens nas diferentes regiões do Chile no período entre 2000 e 2017.

Método. Estudo ecológico populacional a partir de registros de mortes por suicídio. As taxas de mortalidade e riscos relativos $(\mathrm{RR})$ de suicídio foram estimados por sexo, idade e região. A tendência e desigualdades geográficas do suicídio foram analisadas por região com o cálculo das medidas absolutas e relativas das desigualdades geográficas.

Resultados. No período entre 2000 e 2017, foram registrados 6.292 suicídios em adolescentes e jovens no Chile. As taxas médias de mortalidade por suicídio no país foram de 8,5, 5,4 e 14,7 por 100.000 habitantes nas faixas etárias de 10-24, 10-19 e 20-24 anos, respectivamente. As taxas de mortalidade por suicídio foram maiores nas regiões de Aisén, Los Lagos, Magallanes e Los Ríos. O risco de suicídio foi maior no sexo masculino ( $R R=3,5)$, em jovens $(R R=2,7)$ e na região de Aisén ( $R R=2,0)$. A taxa média nacional na faixa etária entre 10 e 24 anos se manteve em 8,5 por 100.000 nos períodos de 2000 a 2008 e de 2009 a 2017. A maior desigualdade geográfica foi vista em homens entre 20 e 24 anos no período de 2000 a 2008.

Conclusão. As taxas de suicídio em adolescentes e jovens do Chile se manteve relativamente estável no período estudado. Indivíduos do sexo masculino têm maior risco de suicídio. Existem desigualdades geográficas entre as regiões do país e as taxas de suicídio são mais elevadas em homens jovens entre 20 e 24 anos. Recomenda-se avaliar e reforçar os programas de prevenção de suicídio em adolescentes e jovens, sobretudo nas regiões e nos grupos populacionais em situação de maior vulnerabilidade.

Palavras-clave Suicídio; adolescente; disparidades nos níveis de saúde; Chile. 